\title{
Characterization of Tm Concentration Dependence of Dosimetric Properties of $\mathrm{NaMgF}_{3}$
}

\author{
Yuma Takebuchi, ${ }^{*}$ Takumi Kato, Daisuke Nakauchi, \\ Noriaki Kawaguchi, and Takayuki Yanagida \\ Division of Materials Science, Nara Institute of Science and Technology (NAIST), \\ Takayama-cho, Ikoma, Nara 630-0192, Japan
}

(Received October 11, 2021; accepted November 17, 2021)

Keywords: $\mathrm{NaMgF}_{3}, \mathrm{OSL}, \mathrm{TSL}$, scintillation

We synthesized Tm-doped $\mathrm{NaMgF}_{3}$ crystals and investigated their scintillation, optically stimulated luminescence (OSL), and thermally stimulated luminescence (TSL) properties. All the crystals showed multiplex luminescence peaks. Judging from the emission wavelength and scintillation decay time constants, $\mathrm{Tm}^{3+}$ acts as a luminescence center in $\mathrm{NaMgF}_{3}$ crystals. The $0.3 \% \mathrm{Tm}$-doped crystal showed the highest scintillation, OSL, and TSL intensities. The $0.3 \%$ Tm-doped crystal showed a linear relationship from 0.01 to $100 \mathrm{mGy}$ for its TSL dose response function. This lower detection limit is comparable to that of some commercial products. In addition, we succeeded in 2D X-ray imaging using the TSL of the crystal.

\section{Introduction}

Dosimetric materials can store absorbed ionizing radiation energy in the form of carriers (electrons and holes). When dosimetric materials are irradiated with ionizing radiation, numerous carriers are generated. The carriers are metastable at localized trapping centers and released by external heat or light stimulation. Then, the detrapped carriers recombine at luminescence centers and emit light. The storage-type luminescence generated by light or heat stimulation is called optically or thermally stimulated luminescence (OSL or TSL, respectively). The luminescence intensity is proportional to the absorbed energy, and we can thus estimate the total amount and distribution of the irradiation dose. Therefore, dosimetric materials are used for personal dose monitoring, ${ }^{(1)}$ medical imaging, ${ }^{(2)}$ radiation therapy, ${ }^{(3,4)}$ and so forth. In addition to OSL and TSL, radio-photoluminescence (RPL) $)^{(5,6)}$ is used for radiation detection. The development of higher performance dosimetric materials for more accurate measurement is still ongoing. ${ }^{(7-12)}$ The required properties of these materials are high luminescence intensity, dose linearity, low fading, and good energy response (small ionization quenching). In addition, high tissue equivalence, i.e., a low effective atomic number $\left(Z_{\text {eff }}\right)$, has been preferred for personal dose monitoring. Moreover, with the development of recent treatment techniques, low- $Z_{\text {eff }}$ materials are also required to accurately estimate the effect of radiation therapy on patients.

${ }^{*}$ Corresponding author: e-mail: takebuchi.yuma.ty1@ms.naist.jp

https://doi.org/10.18494/SAM3685 
$A B \mathrm{~F}_{3}$ ( $A$ : monovalent, $B$ : divalent cation) fluoroperovskite materials generally have a high band gap, ${ }^{(13)}$ high thermal and mechanical stability, and low hygroscopicity. There are some previous works on the dosimetric properties of $A B \mathrm{~F}_{3} \cdot{ }^{(14-16)}$ In particular, Ce-doped $\mathrm{KMgF}_{3}$ has been reported to have high sensitivity as much as 40 times that of the commercial dosimetric material $\mathrm{C}$-doped $\mathrm{Al}_{2} \mathrm{O}_{3} \cdot{ }^{(17)}$ However, $\mathrm{K}$ contains radioisotope ${ }^{40} \mathrm{~K}$ and has intrinsic radioactivity. Therefore, alternative elements that do not contain radioisotopes are preferred. $\mathrm{NaMgF}_{3}$ can be used without considering noise from radioisotopes unlike $\mathrm{KMgF}_{3}$. Moreover, $\mathrm{NaMgF}_{3}$ has high tissue equivalence because of its lower $Z_{\text {eff }}(10.4)$ than that of $\mathrm{KMgF}_{3}(14.9)$ and some commercial products such as $\mathrm{C}$-doped $\mathrm{Al}_{2} \mathrm{O}_{3}$ (11.3) and Ag-doped phosphate glass (12.4). We previously reported the OSL properties of $0.1 \% \mathrm{Tm}$-doped $\mathrm{NaMgF}_{3}{ }^{(18)}$ but its sensitivity is insufficient for practical use. Detailed studies of Tm-doped $\mathrm{NaMgF}_{3}$, including its Tm concentration dependence and TSL properties, would improve its performance and help in the search for new materials with desirable dosimetric properties.

In this study, we synthesized $\mathrm{NaMgF}_{3}$ crystals with various concentrations of $\mathrm{Tm}$ and investigated the Tm concentration dependence of the OSL and TSL properties. Scintillation, which is instantaneous radiation-induced luminescence, properties were also evaluated because the relationship between storage-type luminescence and scintillation has recently been reported, ${ }^{(19,20)}$ although these phenomena were previously regarded as independent. Thus, the evaluation of both phenomena is important to understand ionizing-radiation-induced luminescence phenomena.

\section{Materials and Methods}

Tm-doped $\mathrm{NaMgF}_{3}$ crystals were obtained by a simple solidification method. The nominal concentrations of $\mathrm{Tm}$ were $0.05,0.1$, and $0.3 \%$. We used $\mathrm{NaF}$ (99.99\%, Wako Pure Chemical Industries, Ltd.), $\mathrm{MgF}_{2}$ (99.99\%, Stella Chemifa Corporation), and $\mathrm{TmF}_{3}$ (99.99\%, Stella Chemifa Corporation) powders as the raw materials. The details of the method and growth conditions are given in our previous study. ${ }^{(18)}$ A polishing machine (BUEHLER, MetaServ 250) was used to polish the wide surface of the obtained crystals. Part of the crystals was crushed into powder for measuring the X-ray diffraction (XRD) pattern using a diffractometer (Rigaku, MiniFlex600).

The X-ray-induced scintillation spectra were measured using our original setup. ${ }^{(21)}$ The bias voltage and tube current were $40 \mathrm{kV}$ and $1.2 \mathrm{~mA}$, respectively. X-ray-induced scintillation decay curves were obtained using our afterglow characterization system. ${ }^{(22)}$

The OSL spectra were measured using a spectrofluorometer (FP8600, Hamamatsu) after X-ray irradiation of $10 \mathrm{~Gy}$. The OSL dose response functions were obtained by integrating the OSL intensity over time. Here, the measurement time was $500 \mathrm{~s}$.

The TSL glow curves were investigated using a TSL reader (TL-2000, NanoGray Inc.). (23) The heating rate was $1{ }^{\circ} \mathrm{C} / \mathrm{s}$. The TSL dose response function of each irradiation dose was obtained by integrating the TSL glow curve. To evaluate both the OSL and TSL dose response functions, the samples were irradiated with X-rays with 10-fold increments in the dose rate; the dose rates for each irradiation dose are shown in Table 1. The measurements were made within 
Table 1

Dose rates for each irradiation dose for OSL and TSL dose response functions.

\begin{tabular}{lccccc}
\hline Irradiation dose $(\mathrm{mGy})$ & 0.01 & 0.1 & 1 and 10 & 100 & 1000 and 10000 \\
\hline Dose rate $(\mathrm{mGy} / \mathrm{h})$ & 6 & 60 & 600 & 6000 & 60000
\end{tabular}

$30 \mathrm{~s}$ after irradiation to avoid fading. The TSL spectra were measured with a CCD-based spectrometer (Ocean Optics, QE Pro) while the crystal was heated by an electric heater (SAKAGUCHI E.H VOC, SCR-SHQ-A). ${ }^{(24)}$ The TSL imaging was carried out using our original setup based on an OSL imaging setup. ${ }^{(25)}$ The samples were irradiated with X-rays through an electric component (operational amplifier). After X-ray irradiation, the samples were placed on a hot plate and heated. The 2D TSL distribution was obtained with a Peltier-cooled CCD camera (BK-54DUV, Bitran Corp.) equipped with an objective lens (PF10545MF-UV, Nikon). The distance between the crystal and the camera was set to $80 \mathrm{~cm}$.

\section{Results and Discussion}

Figure 1 shows a photograph of the synthesized crystals after polishing. We obtained translucent crystals with $10 \mathrm{~mm}$ diameter and $1 \mathrm{~mm}$ thickness. The weights of the $0.05,0.1$, and $0.3 \%$ Tm-doped crystals were $373.4,377.8$, and $386.2 \mathrm{mg}$, respectively. Figure 2 depicts the XRD patterns of the crystals. For the 0.05 and $0.1 \%$ Tm-doped crystals, the XRD patterns are identical with the reference pattern (COD.9001612). For the 0.3\% Tm-doped crystal, however, a $\mathrm{TmF}_{3}$ phase was observed at $28^{\circ}$. Therefore, we judged that the $\mathrm{NaMgF}_{3}$ crystal can be synthesized when the Tm concentration is $0.1 \%$ or less using our method. Furthermore, no peak shift was observed; therefore, Tm was probably located at an interstitial.

Figure 3 shows the scintillation spectra of the crystals. All the crystals show scintillation peaks at 290, 345, 360, 450, 470, 510, and $650 \mathrm{~nm}$, in good agreement with other works on Tmdoped materials, ${ }^{(26-28)}$ and the intensity increased with increasing concentration of $\mathrm{Tm}$. The scintillation decay curves and the obtained decay time constants are illustrated in Fig. 4. The decay curves are well fitted by the sum of two exponential functions. The first component $\left(\tau_{1}\right)$ is on the order of $100 \mu \mathrm{s}$, which is a typical value of the $4 \mathrm{f}-4 \mathrm{f}$ transitions of $\mathrm{Tm}^{3+} .(29,30)$ The second component $\left(\tau_{2}\right)$ is likely to be due to some kind of defect. Judging from the emission wavelength and decay time constant, the luminescence is considered to originate from the $4 \mathrm{f}-4 \mathrm{f}$ transitions of $\mathrm{Tm}^{3+}$.

Figure 5 shows the OSL spectra of the crystals after X-ray irradiation of $10 \mathrm{~Gy}$. Here, the stimulation wavelength was $500 \mathrm{~nm}$. OSL peaks at 360, 450, and $470 \mathrm{~nm}$ were observed in all the samples. The luminescence wavelengths were consistent with the scintillation spectra, and the origin of the luminescence was considered to be the $4 \mathrm{f}-4 \mathrm{f}$ transitions of $\mathrm{Tm}^{3+}$. Figure 6 shows the OSL dose response functions of the crystals when the stimulation and monitoring wavelengths were 470 and $360 \mathrm{~nm}$, respectively. The inset shows the OSL dose response functions of the $0.1 \%$ Tm-doped crystal at different monitoring wavelengths. Although the luminescence intensity at $450 \mathrm{~nm}$ is higher than that at $360 \mathrm{~nm}$ for $500 \mathrm{~nm}$ stimulation, a higher 


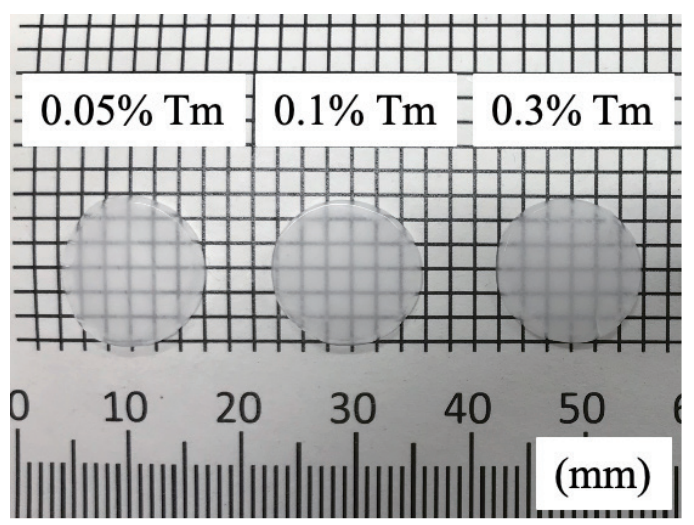

Fig. 1. Photograph of the Tm-doped $\mathrm{NaMgF}_{3}$ crystals.

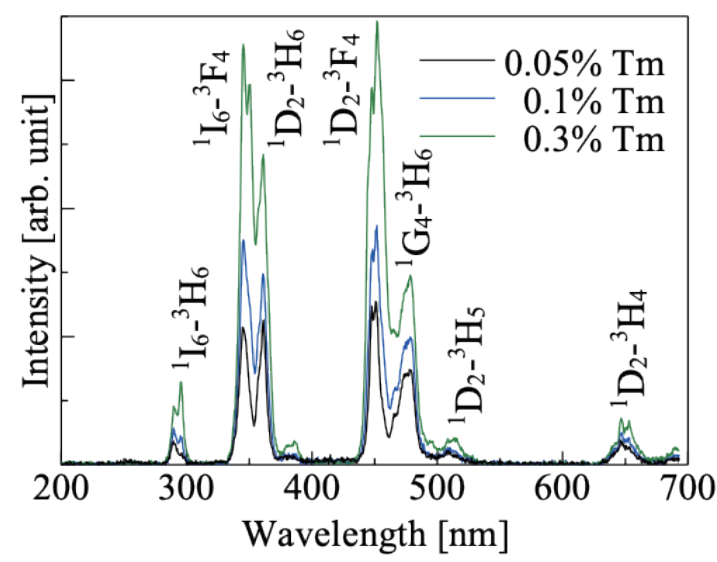

Fig. 3. (Color online) X-ray-induced scintillation spectra of the Tm-doped $\mathrm{NaMgF}_{3}$ crystals. The intensities were corrected by the sample weight.

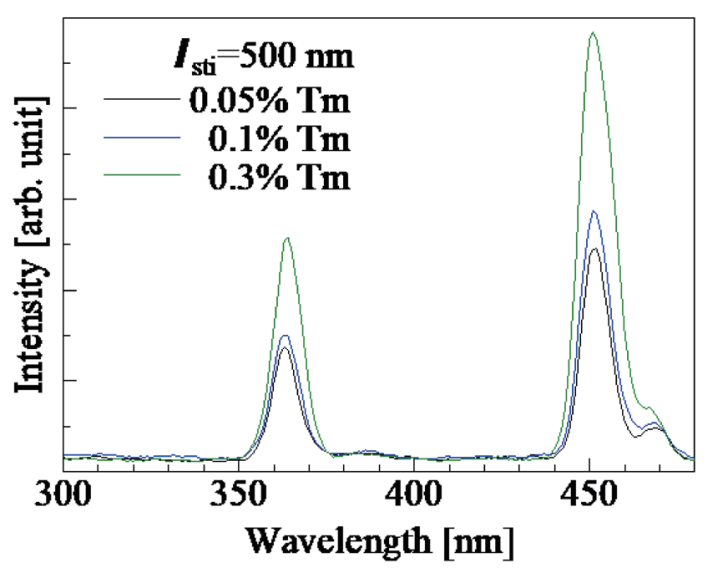

Fig. 5. (Color online) OSL spectra of the Tm-doped $\mathrm{NaMgF}_{3}$ crystals after X-ray irradiation of $10 \mathrm{~Gy}$. The stimulation wavelength was $500 \mathrm{~nm}$. The intensities were corrected by the sample weight.

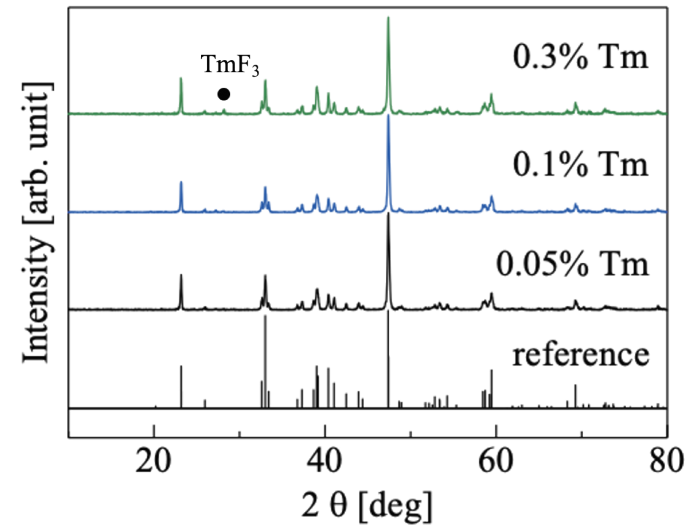

Fig. 2. (Color online) XRD patterns of the Tmdoped $\mathrm{NaMgF}_{3}$ crystals and reference (COD.9001612).

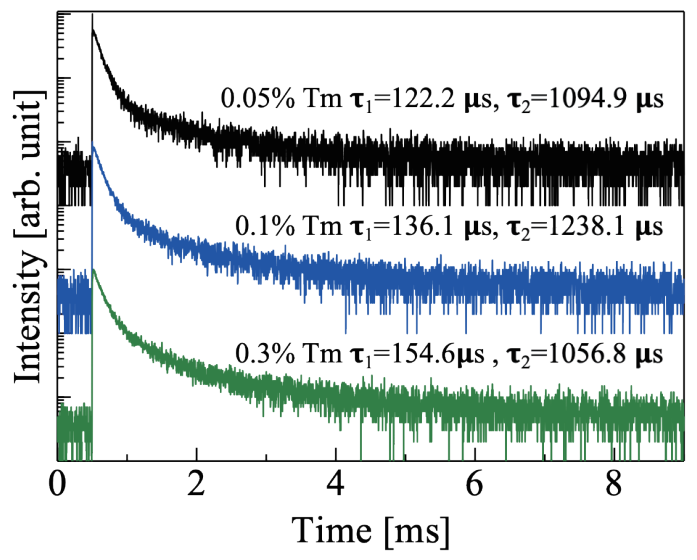

Fig. 4. (Color online) X-ray-induced scintillation decay curves of the Tm-doped $\mathrm{NaMgF}_{3}$ crystals.

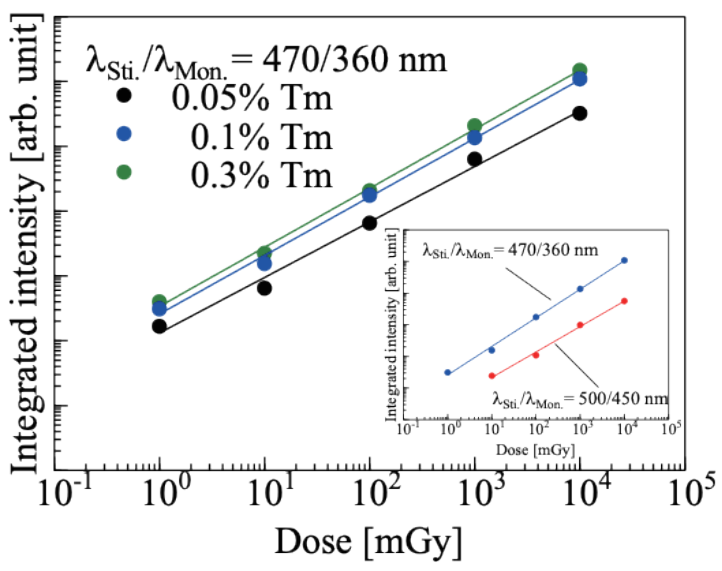

Fig. 6. (Color online) OSL dose response functions of the Tm-doped $\mathrm{NaMgF}_{3}$ crystals. The stimulation and monitoring wavelengths were 470 and $360 \mathrm{~nm}$, respectively. The intensities were corrected by the sample weight. The inset shows the OSL dose response functions of the $0.1 \% \mathrm{Tm}$-doped crystal at different stimulation and monitoring wavelengths. 
intensity was obtained by a shorter stimulation wavelength, similarly to Tm-doped $\mathrm{KMgF}_{3},{ }^{(15)}$ and the luminescence intensity for $\lambda_{\text {sti. }} \cdot \lambda_{\text {mon. }}=470 / 360 \mathrm{~nm}$ is higher than that for $\lambda_{\text {sti. }} / \lambda_{\text {mon. }}=500 / 450 \mathrm{~nm}$. All the crystals showed a linear relationship in the range of $1 \mathrm{mGy}$ to $10 \mathrm{~Gy}$. The intensity increased with increasing Tm concentration, as with the scintillation.

Figure 7 exhibits the TSL glow curves of the crystals. Tm-doped crystals show two peaks at around 80 and $180{ }^{\circ} \mathrm{C}$. According to previous works, some fluoroperovskite materials exhibit two glow peaks at similar temperatures regardless of the combination of the cation and activator; ${ }^{(15,31,32)}$ therefore, the trapping centers may be related to the fluoroperovskite structure. However, further investigation is required to characterize the trapping centers. The highest TSL intensity was obtained from the $0.3 \%$ Tm-doped crystal as well as the highest scintillation and OSL intensities, despite an impurity phase generally having undesired effects on luminescence properties. As one of the reasons, it is considered that the absorption dose was increased by the doping of Tm, which is a heavier element than those in the host material. Therefore, the positive effect of increasing the absorption dose outweighed the negative effect of the impurity phase. In addition, the order of the luminescence intensity among the samples with different doping concentrations is the same for scintillation, OSL, and TSL. However, the dependence (intensity ratio) is different for each luminescence. The difference in the intensity ratio between scintillation and storage luminescence (OSL and TSL) is considered to be due to the luminescence mechanism: scintillation is instantaneous luminescence by carriers that are not trapped at trapping centers, whereas storage luminescence uses trapped carriers. Furthermore, while TSL releases carriers with multiple levels simultaneously, OSL releases only carriers of a specific level corresponding to the stimulation wavelength. Therefore, scintillation, TSL, and OSL have different dependences on the trapping centers. This is thought to have resulted in the difference in the intensity ratio.

Figure 8 shows the TSL spectra of the crystals heated at $80^{\circ} \mathrm{C}$ after X-ray irradiation of $1 \mathrm{~Gy}$. All the crystals showed identical emission peaks to the scintillation spectra. Thus, TSL is also considered to originate from the $4 \mathrm{f}-4 \mathrm{f}$ transitions of $\mathrm{Tm}^{3+}$.

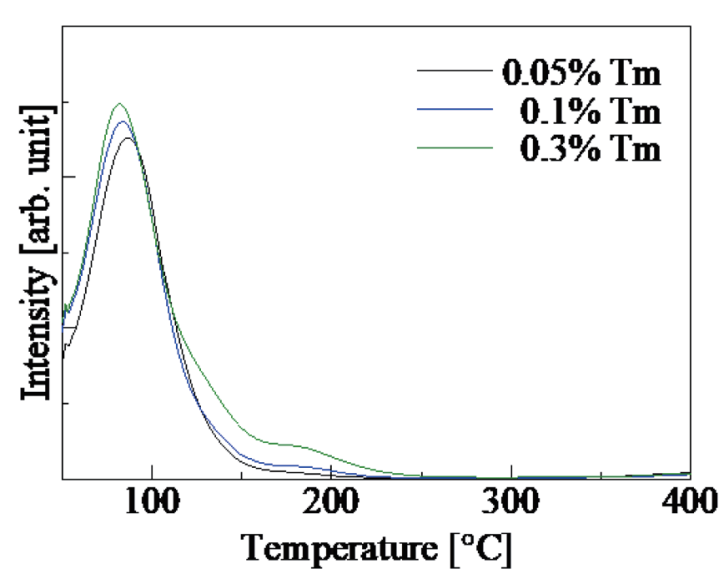

Fig. 7. (Color online) TSL glow curves of the Tm-doped $\mathrm{NaMgF}_{3}$ crystals after X-ray irradiation of $100 \mathrm{mGy}$. The intensities were corrected by the sample weight.

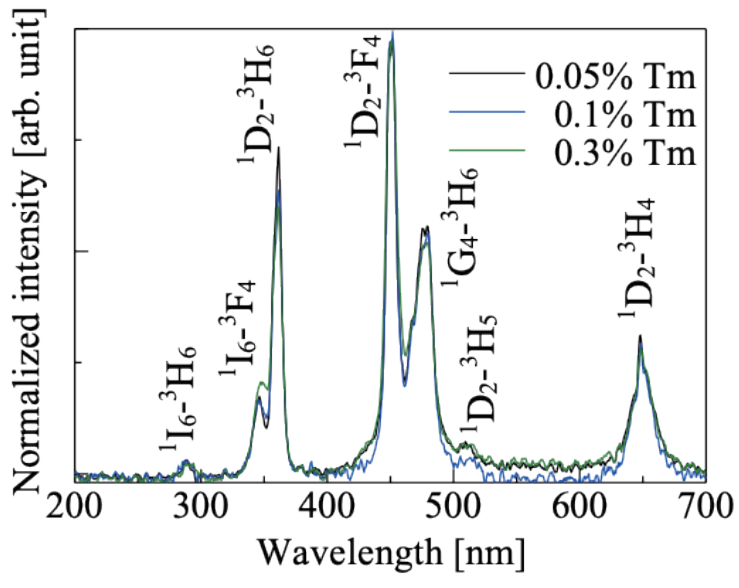

Fig. 8. (Color online) TSL spectra of the Tm-doped $\mathrm{NaMgF}_{3}$ crystals heated at $80{ }^{\circ} \mathrm{C}$ after X-ray irradiation of $1 \mathrm{~Gy}$. 


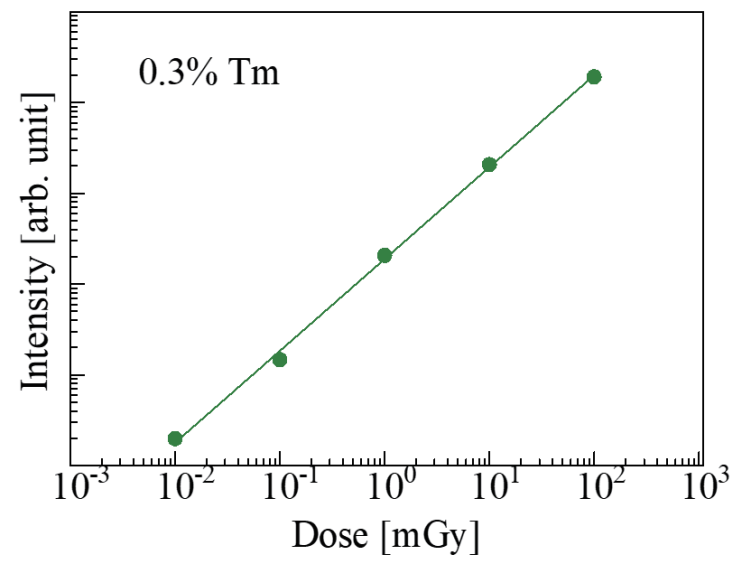

Fig. 9. (Color online) TSL dose response function of the $0.3 \%$ Tm-doped $\mathrm{NaMgF}_{3}$ crystal.

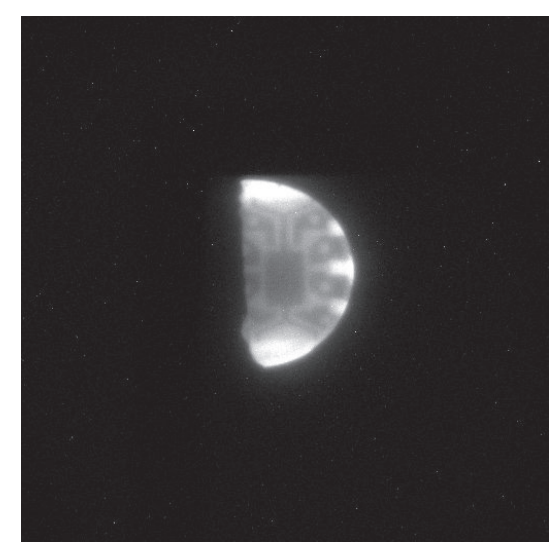

Fig. 10. X-ray image of the electrical component of the $0.3 \% \mathrm{Tm}$-doped $\mathrm{NaMgF}_{3}$ crystal taken by TSL.

Figure 9 depicts the TSL dose response function of the $0.3 \%$ Tm-doped crystal, which showed the highest TSL intensity in Fig. 7. The crystal shows the linear relationship under X-ray irradiation between 0.01 and $100 \mathrm{mGy}$. The crystal is expected to show a wider linear range because $0.01 \mathrm{mGy}$ is the minimum dose in our setup due to the natural environmental dose, and saturation of the detector occurred over $100 \mathrm{mGy}$. The sensitivity of TSL is higher than that of OSL and comparable to that of some commercial personal dose monitoring devices (e.g., C-doped $\mathrm{Al}_{2} \mathrm{O}_{3}$, Nagase Landauer). For both OSL and TSL, multiplex luminescence peaks were observed; however, the OSL dose response functions monitored only a single emission peak, which probably caused an underestimation of the luminescence intensity. Therefore, if all the multiple luminescence peaks can be monitored in OSL dose response functions, improved OSL sensitivity can be expected. Furthermore, since the OSL intensity also depends on the stimulation photon flux and the photoionization cross section, ${ }^{(33)}$ a higher OSL sensitivity is expected by using laser stimulation instead of a practically used xenon lamp.

Figure 10 shows an X-ray image of an operational amplifier taken using the $0.3 \%$ Tm-doped crystal. We can clearly observe the image through the epoxy encapsulation. Because the legs with $1.0 \mathrm{~mm}$ width were clearly separated, the spatial resolution surpassed $1.0 \mathrm{~mm}$. To put our sample into practical use, improvement of the spatial resolution by an order of magnitude is desirable. ${ }^{(34)}$ Achieving more homogeneous crystals and optimizing the thickness will result in higher spatial resolution. Therefore, $\mathrm{Tm}$-doped $\mathrm{NaMgF}_{3}$ is a good candidate for not only personal dosimetry but also 2D dosimetry.

\section{Conclusions}

We have investigated the Tm concentration dependence of the radiation-induced luminescence properties of Tm-doped $\mathrm{NaMgF}_{3}$. All the crystals exhibited luminescence due to the $4 \mathrm{f}-4 \mathrm{f}$ transitions of $\mathrm{Tm}^{3+}$. The $0.3 \% \mathrm{Tm}$-doped $\mathrm{NaMgF}_{3}$ crystal indicated high sensitivity to TSL from a dose of $0.01 \mathrm{mGy}$, which is comparable to that of some commercial products. In addition, we demonstrated the possibility of 2D dosimetry using the crystal. Therefore, the Tm- 
doped $\mathrm{NaMgF}_{3}$ crystal is promising as a new dosimetric material with a high tissue equivalent. There was a positive correlation between scintillation and storage luminescence intensities, and the luminescence intensity of scintillation, OSL, and TSL increased with the concentration of $\mathrm{Tm}$ within the range examined in this study. The synthesis of higher quality crystals with a higher concentration of Tm without an impurity phase is expected to lead to improved dosimetric properties. Furthermore, we intend to estimate the fading properties of Tm-doped $\mathrm{NaMgF}_{3}$ crystal, which are important for practical use in future work.

\section{Acknowledgments}

This work was supported by Grants-in-Aid for Scientific Research B (19H03533, 21H03733, and 21H03736) and Early-Career Scientists (20K15026 and 20K20104) from the Japan Society for the Promotion of Science. The Cooperative Research Project of the Research Center for Biomedical Engineering, Yashima Environment Technology Foundation, Okura Kazuchika Foundation, and Hitachi Metals-Materials Science Foundation are also acknowledged.

\section{References}

1 B. C. Bhatt and M. S. Kulkarni: Int. J. Lumin. Appl. 3 (2013) 6.

2 H. Nanto, A. Nishimura, M. Kuroda, Y. Takei, Y. Nakano, T. Shoji, T. Yanagita, and S. Kasai: Nucl. Instrum. Methods Phys. Res., Sect. A 580 (2007) 278.

3 A. Viamonte, L. A. R. da Rosa, L. A. Buckley, A. Cherpak, and J. E. Cygler: Med. Phys. 35 (2008) 1261.

4 K. Watanabe, A. Yamazaki, K. Nakahashi, H. Miyamae, A. Uritani, and E. Ariga: Radiat. Meas. 55 (2013) 64.

5 T. Yamamoto, Y. Yanagida-Miyamoto, T. Iida, and H. Nanto: Radiat. Meas. 136 (2020) 106363.

6 G. Okada, K. Hirasawa, T. Yanagida, and H. Nanto: Sens. Mater. 33 (2021) 2117.

7 T. Kato, D. Shiratori, M. Iwao, H. Takase, D. Nakauchi, N. Kawaguchi, and T. Yanagida: Sens. Mater. 33 (2021) 2163.

8 T. Kato, D. Nakauchi, N. Kawaguchi, and T. Yanagida: Sens. Mater. 32 (2020) 1411.

9 N. Kawaguchi, G. Okada, Y. Futami, D. Nakauchi, T. Kato, and T. Yanagida: Sens. Mater. 32 (2020) 1419.

10 H. Kimura, T. Kato, D. Nakauchi, N. Kawaguchi, and T. Yanagida: Sens. Mater. 33 (2021) 2187.

11 D. Shiratori, T. Kato, D. Nakauchi, N. Kawaguchi, and T. Yanagida: Sens. Mater. 33 (2021) 2171.

12 R. Oh, S. Yanagisawa, H. Tanaka, T. Takata, G. Wakabayashi, M. Tanaka, N. Sugioka, Y. Koba, and K. Shinsho: Sens. Mater. 33 (2021) 2129.

13 A. V. Gektin, I. M. Krasovitskaya, and N. V. Shiran: Radiat. Meas. 29 (1998) 337.

14 B. Henke, U. Rogulis, and S. Schweizer: Radiat. Meas. 43 (2008) 319.

15 L. Camargo, L. P. Cruz, E. Cruz-Zaragoza, S. M. Ovalle, and J. Marcazzó: Appl. Radiat. Isot. 141 (2018) 219.

16 D. J. Daniel, O. Annalakshmi, U. Madhusoodanan, and P. Ramasamy: J. Rare Earths 32 (2014) 496.

17 N. J. M. Le Masson, A. J. J. Box, C. W. E. Van Eijk, C. Furetta, and J. P. Chaminade: Radiat. Prot. Dosim. 100 (2002) 229.

18 Y. Takebuchi, H. Fukushima, T. Kato, D. Nakauchi, N. Kawaguchi, and T. Yanagida: Sens. Mater. 32 (2020) 1405.

19 T. Yanagida: J. Lumin. 169 (2016) 544.

20 Y. Takebuchi, H. Fukushima, T. Kato, D. Nakauchi, N. Kawaguchi, and T. Yanagida: Jpn. J. Appl. Phys. 59 (2020) 052007.

21 T. Yanagida, K. Kamada, Y. Fujimoto, H. Yagi, and T. Yanagitani: Opt. Mater. 35 (2013) 2480.

22 T. Yanagida, Y. Fujimoto, T. Ito, U. Kor, and K. Mori: Appl. Phys. Express 7 (2014) 6.

23 T. Yanagida, Y. Fujimoto, N. Kawaguchi, and S. Yanagida: J. Ceram. Soc. Jpn. 121 (2013) 988.

24 G. Okada, T. Kato, D. Nakauchi, K. Fukuda, and T. Yanagida: Sens. Mater. 28 (2016) 897.

25 G. Okada, K. Fukuda, S. Kasap, and T. Yanagida: Photonics 3 (2016) 23.

26 N. Kawano, D. Nakauchi, K. Fukuda, G. Okada, N. Kawaguchi, and T. Yanagida: Jpn. J. Appl. Phys. 57 (2018) 102401. 
27 T. Kato, D. Nakauchi, N. Kawaguchi, and T. Yanagida: Opt. Mater. 106 (2020) 110028.

28 M. Akatsuka, D. Nakauchi, T. Kato, N. Kawaguchi, and T. Yanagida: Sens. Mater. 32 (2020) 1373.

29 P. Kantuptim, M. Akatsuka, D. Nakauchi, T. Kato, N. Kawaguchi, and T. Yanagida: J. Alloys Compd. 847 (2020) 156542.

30 H. Fukushima, D. Nakauchi, T. Kato, N. Kawaguchi, and T. Yanagida: Radiat. Meas. 133 (2020) 106280.

31 G. Kitis, C. Furetta, C. Sanipoli, and A. Scacco: Radiat. Prot. Dosim. 65 (1996) 93.

32 E. Habibi, M. Zahedifar, and E. Sadeghi: J. Lumin. 237 (2021) 118173.

33 E. G. Yukihara and S. W. S. McKeever: Phys. Med. Biol. 53 (2008) R351.

34 G. Fiksel, F. J. Marshall, C. Mileham, and C. Stoeckl: Rev. Sci. Instrum. 83 (2012) 086103. 